\title{
A Low-Cost Wind Generator System with a Permanent Magnet Synchronous Generator and Diode Rectifiers
}

\author{
Shinji Kato ${ }^{1}$, Yoshitaka Inui ${ }^{2}$, Masakazu Michihira ${ }^{3 *}$, and Akira Tsuyoshi ${ }^{3 * *}$ \\ ${ }^{1}$ Department of Electrical Engineering, Kobe City College of Technology \\ 8-3, Gakuenhigashi-machi, Nishi-ku, Kobe, 651-2194, Japan \\ Phone: +81-78-795-3238, Fax: +81-78-795-3314, e-mail: kato@kobe-kosen.ac.jp \\ ${ }^{2}$ Department of Electrical and Electronic Eigineering, Toyohashi University of Technology \\ e-mail: inui@eee.tut.ac.jp \\ $3 *, 3 * *$ Department of Electrical Engineering, Kobe City College of Technology \\ e-mail: michi@kobe-kosen.ac.jp ${ }^{3 *}$, tsuyoshi@kobe-kosen.ac.jp ${ }^{3 * *}$
}

\begin{abstract}
A low-cost wind generator system for small- or medium-scale wind generator is proposed. The system consists of a permanent magnet synchronous generator (PMSG) with two sets of the three-phase stator windings, i.e. six-phase stator winding, two three-phase diode rectifiers, a boost chopper, and a three-phase pulse-width modulation (PWM) inverter. Since two sets of three-phase stator windings are spatially shifted by 30 electrical degrees, the system can cancel out the dominant harmonic magnetomotive forces (MMF) produced by the harmonic currents of diode rectifiers. The simulation and experimental results show that the proposed system can significantly reduce the pulsation of the developed torque and the DC current by more than $60 \%$ in comparison with the conventional system consisting of a three-phase PMSG and a three-phase diode rectifier. Therefore, the proposed system can use a smaller smoothing reactor in the boost chopper, so that the lower initial cost can be realized, and it is suitable for small- or medium-scale wind generator systems.
\end{abstract}

\section{Key words}

Permanent magnet synchronous generator, dual three-phase stator windings, diode rectifier, renewable energy system

\section{Introduction}

Wound-rotor induction generators have advantages of a low-cost, a robustness, and a fractional power rating of power converters. Therefore, the generators are often used in large-scale wind generator systems. However, the generators are seldom used in smallor medium-scale wind generator systems in recent years because of the poor efficiency and power factor.
Thus, PMSGs have been used in small- or mediumscale wind generator systems thanks to the reduction of the permanent magnet material cost. The PMSG needs no excitation current and thus the higher efficiency and power factor can be realized in comparison with the wound-rotor induction generator. As a result, the machine size of the PMSG is smaller than that of the wound-rotor induction generator provided that the power rating of these generators is same[1]. Additionally, the PMSG needs no requirement for the maintenance of brushes and slip-rings. However, the power rating of power converters is the same as the generated power. Hence, the initial cost of the system is more expensive than that of the system consisting of the wound-rotor induction generator.

This paper proposes a low-cost wind generator system consisting of the PMSG with two sets of three-phase stator windings and diode rectifiers in order to reduce the initial cost. The simulation and experimental results show that the proposed system can dramatically reduce the pulsation of the torque and the DC current.

\section{Conventional System}

A PWM converter is often used for the control of the generated power and for the conversion of the generated $\mathrm{AC}$ power into the $\mathrm{DC}$ power in the wind generator system using PMSGs. The power rating of the PWM converter is the same as the generated power as mentioned above. This demands the more expensive initial cost in comparison with the wind generator system using the wound-rotor induction generator. In order to use low-cost power converters, the wind generator system consisting of a three-phase PMSG, a diode rectifier, a boost chopper, and a PWM inverter has been proposed as shown in Fig. 1[2]. 


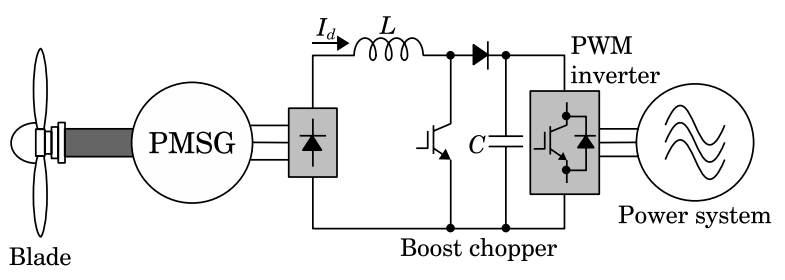

Fig. 1: Conventional system for wind generator.

The diode rectifier converts the generated $\mathrm{AC}$ power, whose frequency depends on the operating speed of the PMSG, into the DC power with no control of semiconductor devices. Therefore, it is able to reduce the cost of the power converter and its controller in comparison with the PWM converter. However, the pulsation appears in the DC current $\left(I_{d}\right)$ and the developed torque since the diode rectifier produces harmonic components of $6 m \pm 1$ ( $m$ :integer) such as fifth and seventh in the stator currents.

The boost chopper controls the generated power and boosts the output voltage of the diode rectifier to the input voltage of the PWM inverter by adjusting a duty-ratio.

The PWM inverter converts the DC power into the three-phase AC power, whose frequency synchronizes the power system, and returns the $\mathrm{AC}$ power to the power system with or without a three-phase transformer.

\section{Proposed System}

The pulsation of the DC current and the developed torque appears in the conventional system due to the harmonic MMF components. Therefore, we propose a novel wind generator system which can reduce the pulsation.

\section{A. System structure}

The proposed system is depicted in Fig. 2. The structure of the system is similar to the conventional system except for the stator windings and the number of diode rectifiers. The PMSG of the system has two sets of three-phase stator windings (stator1 and stator2) with a phase shift angle of 30 electrical degrees so as to reduce the harmonic MMFs produced by diode rectifiers [3]. In spite of two sets of three-phase stator windings, the PMSG demands no special design calculation, and it can be built by rewinding the stator of the widely used three-phase PMSG.

\section{B. Principle of the MMF cancellation}

The principle of the MMF cancellation in the proposed system is described here. The following assumptions are made:

1. Two sets of stator windings are sinusoidally dis-

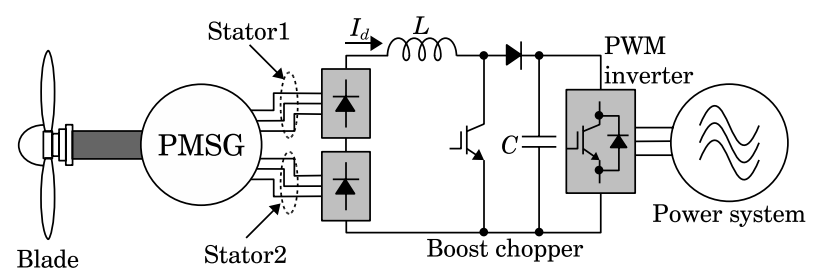

Fig. 2: Proposed system for wind generator.

tributed, i.e. the MMF has no space harmonics.

2. Two neutrals of dual three-phase stator windings are isolated with each other.

Therefore, with respect to the mechanical angle $\theta$, the MMF produced by the current in the air-gap is written as

$$
F=N I \sin \theta
$$

where $N$ is the number of turns and $I$ is the amplitude of the current.

First, we describe MMFs produced by the fifth harmonic stator currents.

The fifth harmonic currents of the stator 1 winding are expressed as follow:

$$
\left\{\begin{array}{l}
i_{a 51}=I_{51} \sin 5 \omega t \\
i_{b 51}=I_{51} \sin 5\left(\omega t-\frac{2}{3} \pi\right) \\
i_{c 51}=I_{51} \sin 5\left(\omega t-\frac{4}{3} \pi\right)
\end{array}\right.
$$

where $I_{51}$ is the amplitude of the fifth harmonic current of the stator1, $\omega$ is the angular velocity of the MMF, and $t$ is the time. Consequently, the MMF produced by the currents of (2) can be written by

$$
\begin{aligned}
F_{51}= & N i_{a 51} \sin \theta+N i_{b 51} \sin \left(\theta-\frac{2}{3} \pi\right) \\
+ & N i_{c 51} \sin \left(\theta-\frac{4}{3} \pi\right) \\
= & N I_{51}\left\{\sin \theta \sin 5 \omega t+\sin \left(\theta-\frac{2}{3} \pi\right) \sin 5\left(\omega t-\frac{2}{3} \pi\right)\right. \\
& \left.\quad+\sin \left(\theta-\frac{4}{3} \pi\right) \sin 5\left(\omega t-\frac{4}{3} \pi\right)\right\} \\
=N I_{51} & {\left[\frac{1}{2}\{\cos (\theta-5 \omega t)-\cos (\theta+5 \omega t)\}\right.} \\
& +\frac{1}{2}\left\{\cos \left(\theta-5 \omega t+\frac{2}{3} \pi\right)-\cos (\theta+5 \omega t)\right\} \\
& \left.+\frac{1}{2}\left\{\cos \left(\theta-5 \omega t-\frac{2}{3} \pi\right)-\cos (\theta+5 \omega t)\right\}\right] \\
=- & \frac{3}{2} N I_{51} \cos (\theta+5 \omega t)
\end{aligned}
$$

where the MMF of $-5 \omega t$ rotates in the clockwise direction, while the MMF of $+5 \omega t$ rotates in the counterclockwise direction. As a result, the MMF of $F_{51}$ rotates in the opposite direction to the fundamental MMF. 
The fifth harmonic stator currents of the stator2 winding are expressed as follow:

$$
\left\{\begin{array}{l}
i_{a 52}=I_{52} \sin 5\left(\omega t-\frac{\pi}{6}\right) \\
i_{b 52}=I_{52} \sin 5\left(\omega t-\frac{\pi}{6}-\frac{2}{3} \pi\right) \\
i_{c 52}=I_{52} \sin 5\left(\omega t-\frac{\pi}{6}-\frac{4}{3} \pi\right)
\end{array}\right.
$$

where $I_{52}$ is the amplitude of the fifth harmonic current of the stator $2, \omega$ is the angular velocity of MMF, and $t$ is the time. Consequently, the MMF produced by the currents of (4) can be written by

$$
\begin{aligned}
& F_{52}= N i_{a 52} \sin \left(\theta-\frac{\pi}{6}\right)+N i_{b 52} \sin \left(\theta-\frac{\pi}{6}-\frac{2}{3} \pi\right) \\
&+N i_{c 52} \sin \left(\theta-\frac{\pi}{6}-\frac{4}{3} \pi\right) \\
&=N I_{52}\{ \sin \left(\theta-\frac{\pi}{6}\right) \sin 5\left(\omega t-\frac{\pi}{6}\right) \\
&+\sin \left(\theta-\frac{\pi}{6}-\frac{2}{3} \pi\right) \sin 5\left(\omega t-\frac{\pi}{6}-\frac{2}{3} \pi\right) \\
&\left.+\sin \left(\theta-\frac{\pi}{6}-\frac{4}{3} \pi\right) \sin 5\left(\omega t-\frac{\pi}{6}-\frac{4}{3} \pi\right)\right\} \\
&=N I_{52}[ {\left[\frac{1}{2}\left\{\cos \left(\theta-5 \omega t+\frac{2}{3} \pi\right)+\cos (\theta+5 \omega t)\right\}\right.} \\
&+ \frac{1}{2}\left\{\cos \left(\theta-5 \omega t-\frac{2}{3} \pi\right)+\cos (\theta+5 \omega t)\right\} \\
&\left.+\frac{1}{2}\{(\cos \theta-5 \omega t)+\cos (\theta+5 \omega t)\}\right] \\
&=\frac{3}{2} N I_{52} \cos (\theta+5 \omega t)
\end{aligned}
$$

where the MMF of $-5 \omega t$ rotates in the clockwise direction, while the MMF of $+5 \omega t$ rotates in the counterclockwise direction. As a result, the MMF of $F_{52}$ rotates in the opposite direction to the fundamental MMF.

From (3) and (5), the resultant MMF of the fifth harmonic component in the air-gap becomes

$$
\begin{aligned}
F_{5} & =F_{51}+F_{52} \\
& =-\frac{3}{2} N I_{m 5} \cos (\theta+5 \omega t)+\frac{3}{2} N I_{m 5} \cos (\theta+5 \omega t) \\
& =0
\end{aligned}
$$

Next, we describe MMFs produced by the seventh harmonic stator currents.

The seventh harmonic currents of the stator1 winding are expressed as follow:

$$
\left\{\begin{array}{l}
i_{a 71}=I_{71} \sin 7 \omega t \\
i_{b 71}=I_{71} \sin 7\left(\omega t-\frac{2}{3} \pi\right) \\
i_{c 71}=I_{71} \sin 7\left(\omega t-\frac{4}{3} \pi\right)
\end{array}\right.
$$

where $I_{71}$ is the amplitude of the seventh harmonic current of the stator1, $\omega$ is the angular velocity of MMF, and $t$ is the time. Therefore, the MMF pro- duced by the currents of (7) can be written by

$$
\begin{aligned}
F_{71}= & N i_{a 71} \sin \theta+N i_{b 71} \sin \left(\theta-\frac{2}{3} \pi\right) \\
& +N i_{c 71} \sin \left(\theta-\frac{4}{3} \pi\right) \\
= & N I_{71}\left\{\sin \theta \sin 7 \omega t+\sin \left(\theta-\frac{2}{3} \pi\right) \sin 7\left(\omega t-\frac{2}{3} \pi\right)\right. \\
& \left.\quad+\sin \left(\theta-\frac{4}{3} \pi\right) \sin 7\left(\omega t-\frac{4}{3} \pi\right)\right\} \\
=N I_{71} & {\left[\frac{1}{2}\{\cos (\theta-7 \omega t)-\cos (\theta+7 \omega t)\}\right.} \\
& +\frac{1}{2}\left\{\cos (\theta-7 \omega t)-\cos \left(\theta+7 \omega t+\frac{2}{3} \pi\right)\right\} \\
& \left.+\frac{1}{2}\left\{\cos (\theta-7 \omega t)-\cos \left(\theta+7 \omega t-\frac{2}{3} \pi\right)\right\}\right] \\
=\frac{3}{2} N I_{71} & \cos (\theta-7 \omega t)
\end{aligned}
$$

where the MMF of $-7 \omega t$ rotates in the clockwise direction, while the MMF of $+7 \omega t$ rotates in the counterclockwise direction. As a result, the MMF of $F_{71}$ rotates in the same direction to the fundamental MMF.

The seventh harmonic currents of the stator 2 winding are expressed as follow:

$$
\left\{\begin{array}{l}
i_{a 72}=I_{72} \sin 7\left(\omega t-\frac{\pi}{6}\right) \\
i_{b 72}=I_{72} \sin 7\left(\omega t-\frac{\pi}{6}-\frac{2}{3} \pi\right) \\
i_{c 72}=I_{72} \sin 7\left(\omega t-\frac{\pi}{6}-\frac{4}{3} \pi\right)
\end{array}\right.
$$

where $I_{72}$ is the amplitude of the seventh harmonic current of the stator $2, \omega$ is the angular velocity of the MMF, and $t$ is the time. Therefore, the MMF produced by the currents of (9) can be written by

$$
\begin{aligned}
& F_{72}=N i_{a 72} \sin \left(\theta-\frac{\pi}{6}\right)+N i_{b 72} \sin \left(\theta-\frac{\pi}{6}-\frac{2}{3} \pi\right) \\
&+N i_{c 72} \sin \left(\theta-\frac{\pi}{6}-\frac{4}{3} \pi\right) \\
&=N I_{72}\left\{\sin \left(\theta-\frac{\pi}{6}\right) \sin 7\left(\omega t-\frac{\pi}{6}\right)\right. \\
& \quad+\sin \left(\theta-\frac{\pi}{6}-\frac{2}{3} \pi\right) \sin 7\left(\omega t-\frac{\pi}{6}-\frac{2}{3} \pi\right) \\
&\left.\quad+\sin \left(\theta-\frac{\pi}{6}-\frac{4}{3} \pi\right) \sin 7\left(\omega t-\frac{\pi}{6}-\frac{4}{3} \pi\right)\right\} \\
&=N I_{72}\left[\frac{1}{2}\left\{-\cos (\theta-7 \omega t)+\cos \left(\theta+7 \omega t+\frac{2}{3} \pi\right)\right\}\right. \\
&+\frac{1}{2}\left\{-\cos (\theta-7 \omega t)+\cos \left(\theta+7 \omega t-\frac{2}{3} \pi\right)\right\} \\
&\left.+\frac{1}{2}\{-\cos (\theta-7 \omega t)+\cos (\theta+7 \omega t)\}\right] \\
&=-\frac{3}{2} N I_{m 7} \cos (\theta-7 \omega t)
\end{aligned}
$$

where the MMF of $-7 \omega t$ rotates in the clockwise direction, while the MMF of $+7 \omega t$ rotates in the counterclockwise direction. As a result, the MMF of $F_{72}$ rotates in the same direction to the fundamental 
MMF.

From (8) and (10), the resultant MMF of the seventh harmonic component in the air-gap becomes

$$
\begin{aligned}
F_{7} & =F_{71}+F_{72} \\
& =\frac{3}{2} N I_{m 7} \cos (\theta-7 \omega t)-\frac{3}{2} N I_{m 7} \cos (\theta-7 \omega t) \\
& =0
\end{aligned}
$$

(6) and (11) show that fifth and seventh harmonic components of the MMF are canceled out by using two sets of three-phase stator windings with the phase-shift angle of 30 electrical degrees. Consequently, the dominant harmonic components of the MMF become $12 n \pm 1$ ( $n$ : integer). This means that the pulsating frequency of the torque becomes higher, and also the width of the pulsating torque becomes smaller.

\section{Simulation and Experiment}

\section{A. Simulation tool}

The conventional and proposed systems have many diodes to convert the generated $\mathrm{AC}$ power into the DC power. Therefore, the circuit topology of those systems is continually changed by the conducting states of diodes, so that it is pretty difficult to simulate those systems by using the equivalent circuit on $d$ and $q$ axes. In this paper, we used the original simulation tool developed by one of the present authors [4] [5]. The original simulation tool adopts the voltage and torque equations on $a, b$, and $c$ axes and thus it is not necessary to derive these equations on $d$ and $q$ axes and to consider the conducting states of diodes.

\section{B. Parameters for simulation and experiment}

Table 1 gives parameters of the PMSG and circuit for the simulation and experiment.

A load resistor $\left(R_{L}\right)$ was connected on a parallel with the output of diode rectifiers instead of the boost chopper and the PWM inverter in order to show the obvious difference in the pulsation of the DC current and the developed torque. Also, the load resistance of the proposed system is a double of the conventional one to generate the same amplitude of the DC current in the both systems.

\section{Simulation and experimental results}

The simulation and experiment of the conventional and proposed systems were carried out at some different operating speeds. The results of simulation and experiment are only presented, due to the space limitation, at an operating speed of 1100 (rpm).
Table 1: Parameters for simulation and experiment.

\begin{tabular}{ll}
\hline \multicolumn{2}{c}{ PMSG parameters } \\
\hline Rated power & $2.2(\mathrm{~kW})$ \\
Stator resistance & $0.36(\Omega)$ \\
$d$-axis inductance & $4.5(\mathrm{mH})$ \\
$q$-axis inductance & $8.5(\mathrm{mH})$ \\
Magnet flux-linkage & $0.09(\mathrm{~Wb})$ \\
Number of poles & 6 \\
Number of stator slots & 36 \\
\hline \multicolumn{2}{c}{ Circuit parameters } \\
\hline Load resistance $\left(R_{L}\right)$ & $12.6(\Omega)$ [Conventional] \\
\multicolumn{2}{c}{$25.3(\Omega)$ [Proposed] }
\end{tabular}

Figs. 3 and 4 show the simulation and experimental results of the stator current and its spectrum in the conventional and proposed system, respectively. These results show a good agreement with each other. From the fast Fourier transform (FFT) analysis, these currents contain a relatively large fifth harmonic content of about $20 \%$, and thus these currents become trapezoidal waveforms. In the proposed system, two sets of three-phase stator windings have the phase shift angle of 30 electrical degrees with each other, so that the stator 2 current lags by 30 degrees to the stator1 current.

The DC currents of the both systems are depicted in Figs. 5 and 6 . Due to the effect of the diode rectifier, the pulsating frequency is $6 f$ where $f=p n$ ( $p$ : number of pole pairs, $n$ : rotational speed) in the conventional system, while that of the proposed system is $12 f$. We can see from the simulation and experimental results that the pulsating frequency of the conventional system is $330(\mathrm{~Hz})$, while that of the proposed system is $660(\mathrm{~Hz})$. Also, the pulsation width in the proposed system is nearly half of the conventional one.

Fig. 7 shows the simulation results of the electromagnetic torque developed by stator currents in both systems. The minus torque means the input torque to the PMSG. Since the pulsating torque is produced by the harmonic components of stator currents, the pulsating frequency is the same as the frequency of the DC current. The proposed system can cancel out the fifth and seventh harmonic components of the MMF, so that the pulsation width can be significantly reduced. The amplitude of the torque in the proposed system is larger than the conventional one because the output voltage of the diode rectifier is double of the conventional system.

Table 2 summarizes the pulsation width in the both systems. In the DC current, the pulsation width of the proposed system is reduced by around $67 \%$. Similarly, the pulsation width of the torque in the proposed system is reduced by around $76 \%$. This significant reduction is brought by the cancellation of the fifth and seventh harmonic components of the MMF. 


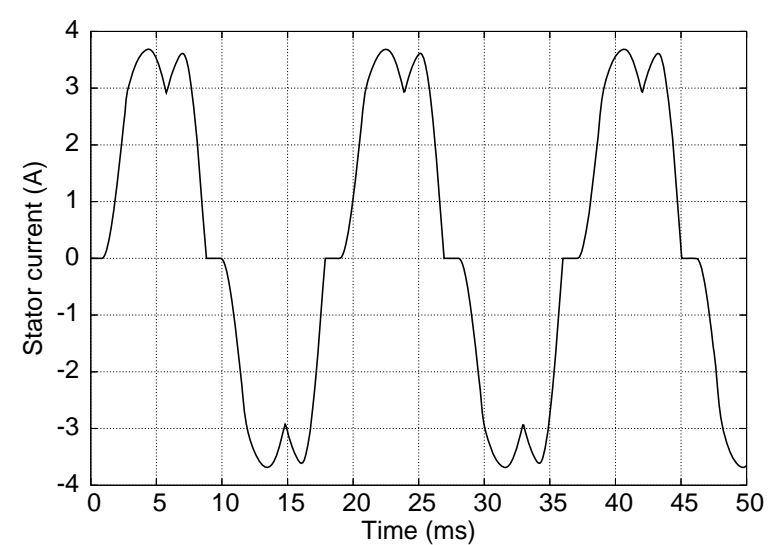

(a) Simulation result.

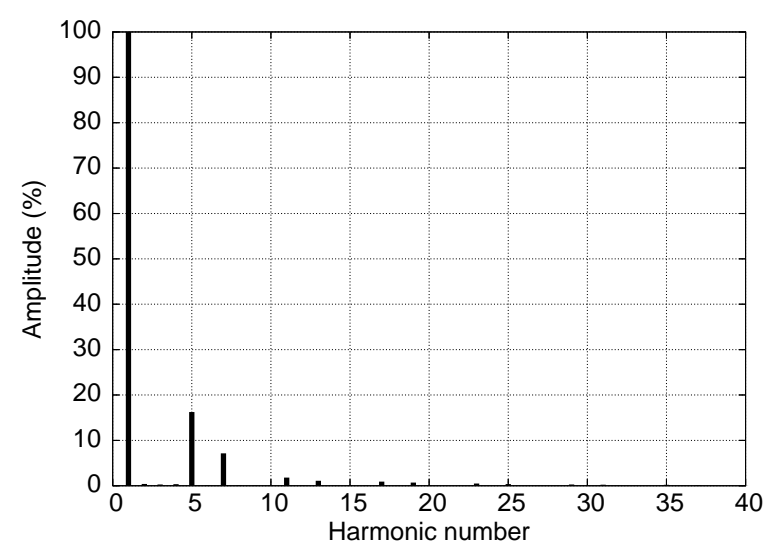

(b) Spectrum of the simulation result.

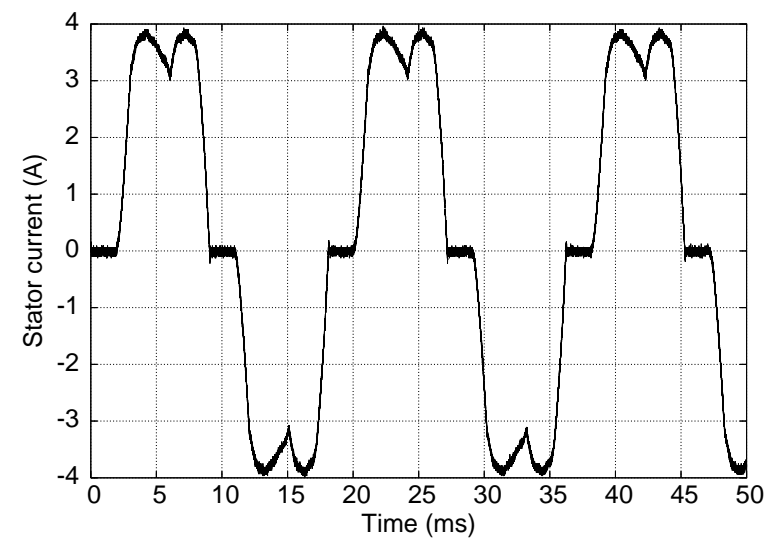

(c) Experimental result.

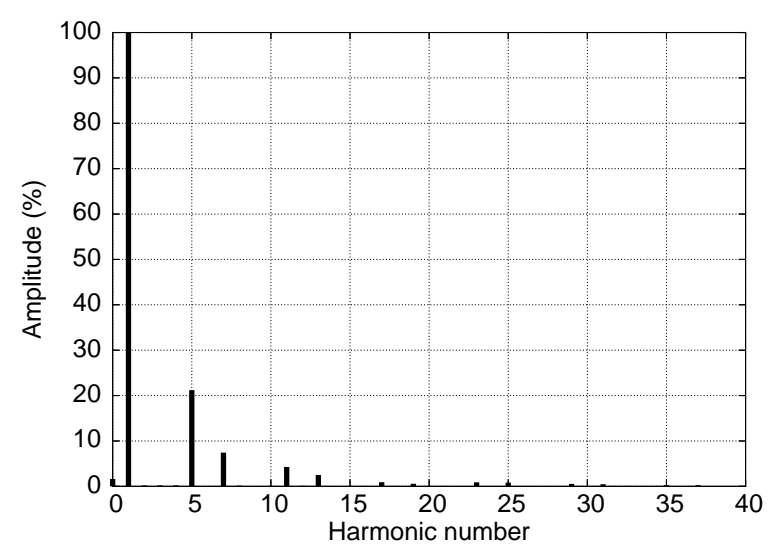

(d) Spectrum of the experimental result.

Fig. 3: Stator currents of the conventional system.

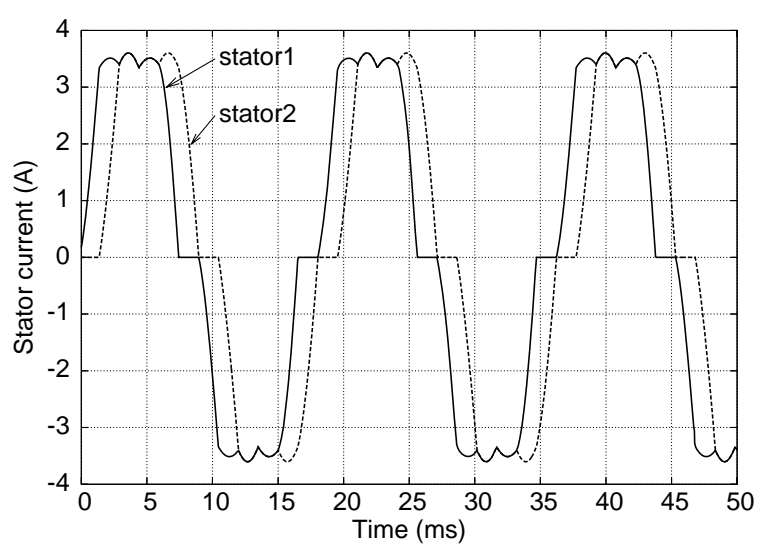

(a) Simulation result.

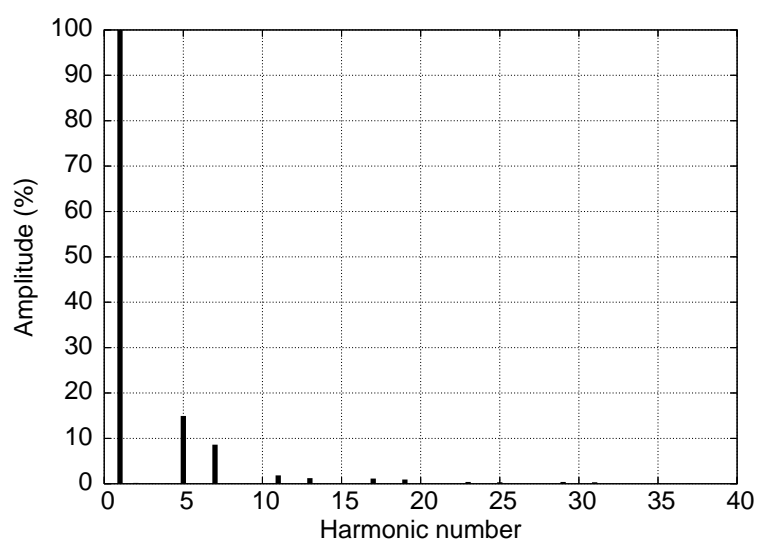

(b) Spectrum of the simulation result.

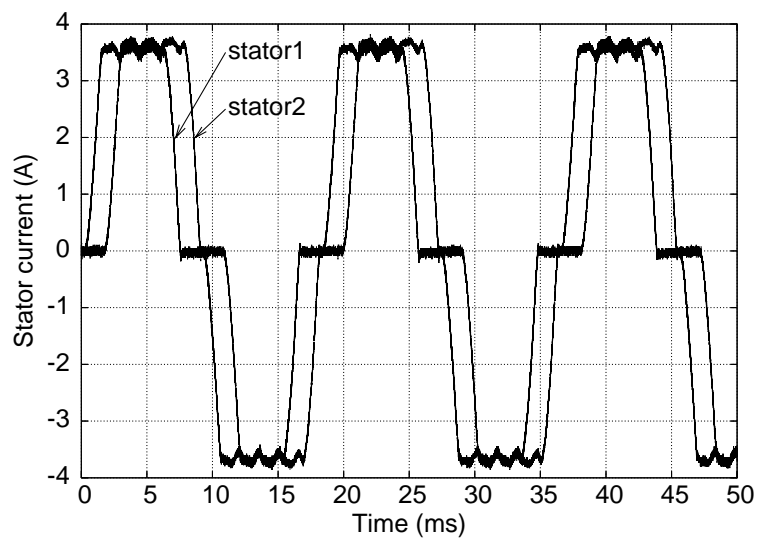

(c) Experimental result.

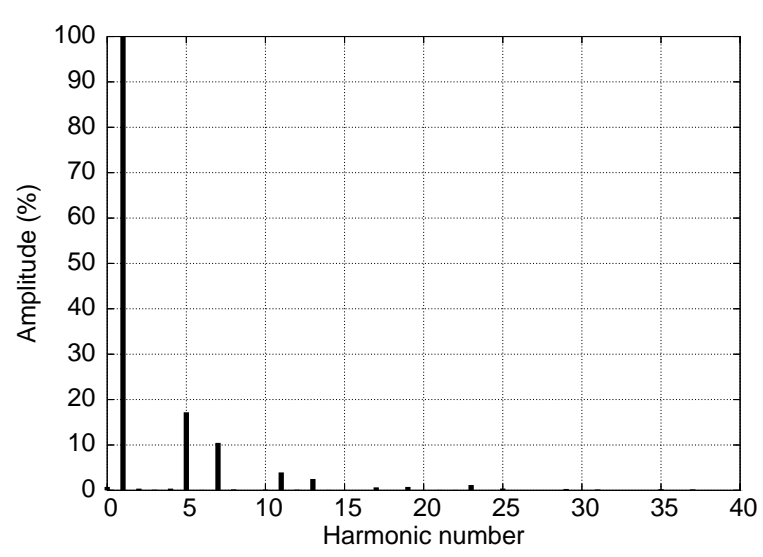

(d) Spectrum of the experimental result.

Fig. 4: Stator currents of the proposed system. 


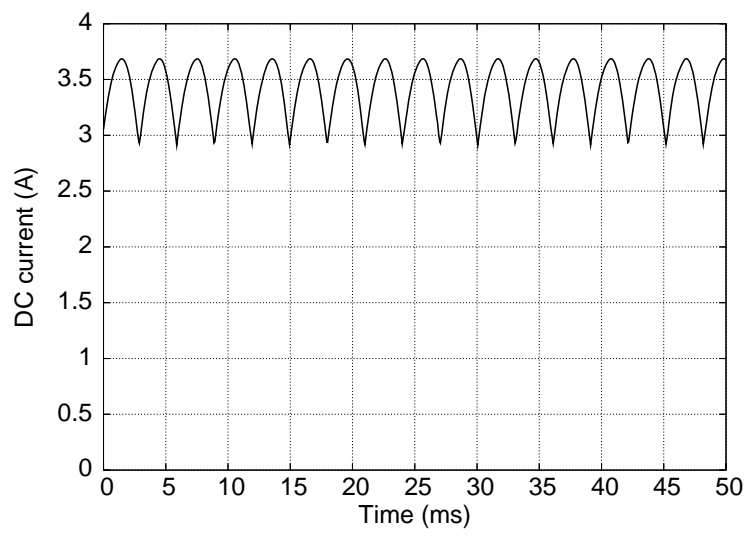

(a) Simulation result.

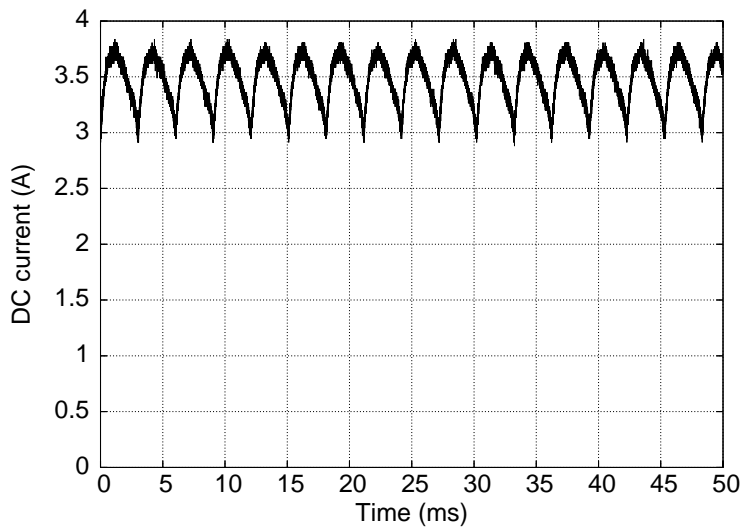

(b) Experimental result.

Fig. 5: DC currents of the conventional system.

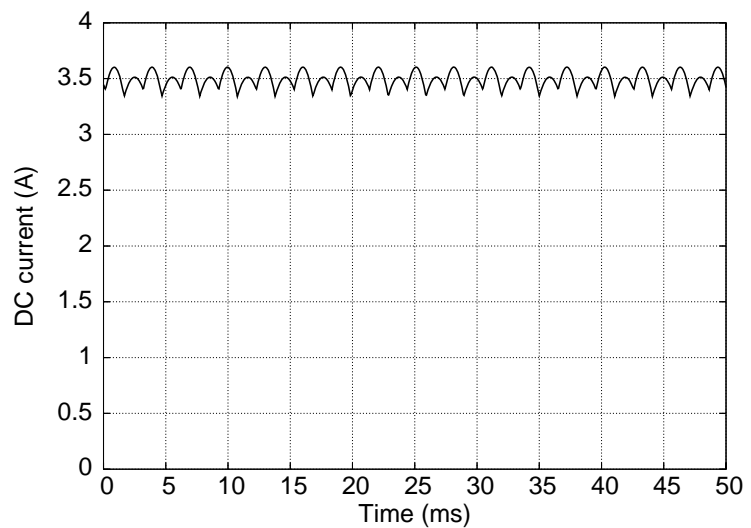

(a) Simulation result.

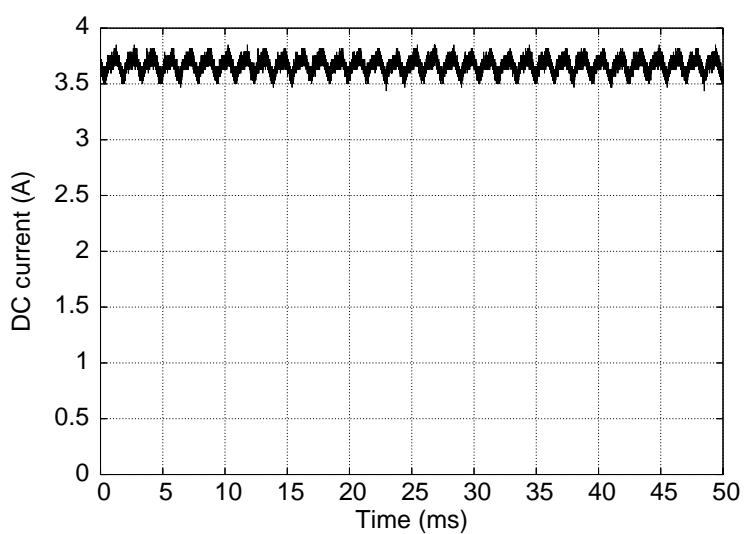

(b) Experimental result.

Fig. 6: DC currents of the proposed system.

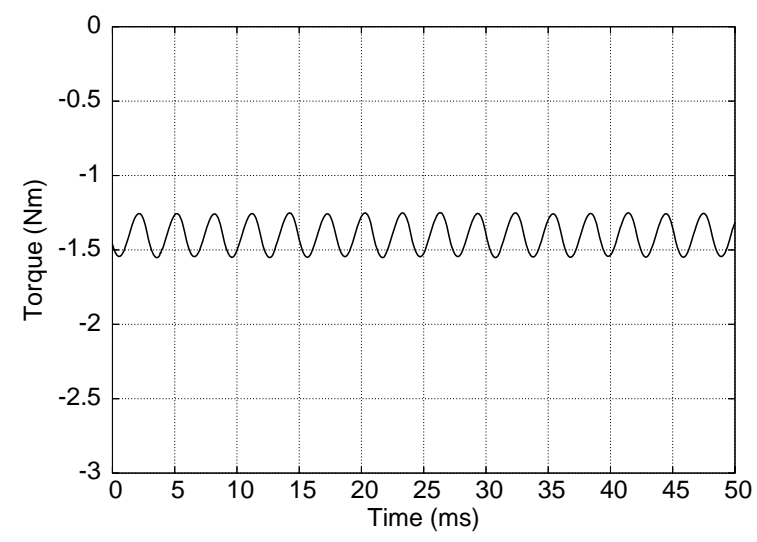

(a) Conventional system.

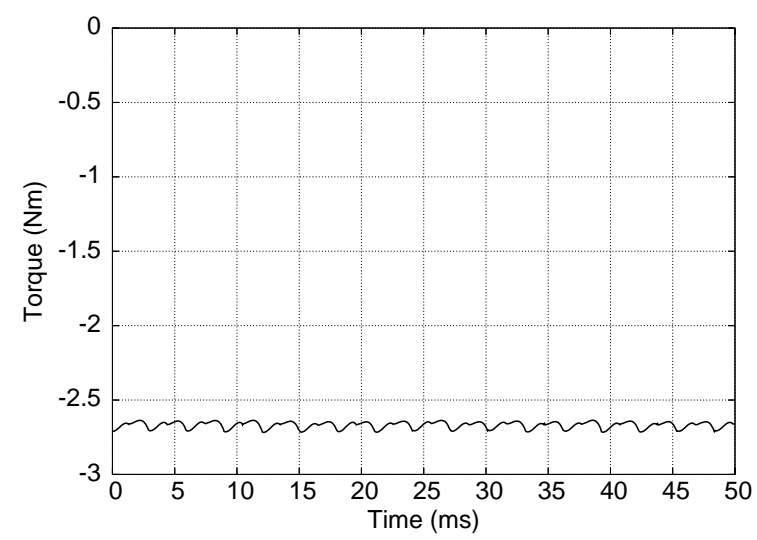

(b) Proposed system.

Fig. 7: Electromagnetic torques in the simulation.

Table 2: Pulsation width of the both systems.

\begin{tabular}{lll} 
& Conventional & Proposed \\
\hline DC current & $0.765(\mathrm{~A})$ & $0.254(\mathrm{~A})$ \\
Torque & $0.291(\mathrm{Nm})$ & $0.071(\mathrm{Nm})$ \\
\hline
\end{tabular}

\section{Conclusions}

A low-cost wind generator system consisting of the PMSG with the six-phase stator winding and diode rectifiers has been proposed. The simulation and experiment of the conventional and proposed systems were carried out. The simulation and experimental results show that the proposed system can dramatically reduce the pulsation in the DC current and torque by more than $60 \%$. Since the proposed system can produce the DC current with less pulsation, the system requires the smaller smoothing reactor in the boot chopper. As a result, the lower initial cost can be realized, and it is suitable for small- or medium-scale wind generator systems.

\section{Acknowledgments}

The authors are grateful to "Hyogo Science and Technology Association" and "Toyohashi University of Technology" for the financial support. 


\section{References}

[1] A. Binder and T. Schneider, "Permanent Magnet Synchronous Generators for Regenerative Energy Conversion - A Survey," in Conf. Rec. 11th European Conference on Power Electronics and Applications, no.761, Sept 2005.

[2] K. Amei, Y. Takayasu, T. Ohji and M. Sakui, "A Maximum Power Control of Wind Generator System Using a Permanent Magnet Synchronous Generator and a Boost Chopper Circuit," Conf. Rec. Power Conversion Conference, pp. 1447-1452, Apr 2002.
[3] S. Kato, N.Hoshi, and K. Oguchi, "Small-Scale Hydropower - Adjustable-Speed Cascaded Induction Generators," IEEE Industry Applications Magazine, Vol. 9, No. 4, pp. 32-38, Jul/Aug 2003.

[4] S. Kato, N. Hoshi, and K. Oguchi, "Analysis of Power Electronics Systems including Cascaded Induction Machines with Modified Nodal Analysis", Proceedings of the Power Conversion ConferenceOsaka, pp. 282-287, Apr 2002.

[5] S. Kato, M. Michihira, and A. Tsuyoshi, "Modeling and Simulation of a Permanent Magnet Synchronous Machine with Six-Phase Stator Winding for Renewable Energy Applications", Proceedings of the 2006 International Conference on Electrical Machines and Systems, Nov 2006. 